\title{
Nanomaterials in art conservation
}

\author{
Piero Baglioni, Emiliano Carretti and David Chelazzi
}

\section{Tackling the degradation of cultural heritage requires a global effort. We call on all material scientists to develop new nanomaterials and methods for the preservation of artwork.}

$\mathrm{T}$ he preservation and transfer of cultural identities and heritage to future generations is everyone's responsibility. A review on the social and economic value of cultural heritage published in 2014 by the European Expert Network on Culture concluded that cultural heritage is a strategic resource for a sustainable Europe: if properly managed, it can enhance social inclusion and cohesion, encourage intercultural dialogue, improve the quality of the immediate living environment, and stimulate tourism ${ }^{1}$. The best way to realize these benefits is to increase access to cultural heritage through digital means and public engagement ${ }^{1}$. However, access is possible only if the original artefacts are properly conserved, stored and displayed. Therefore, developing new conservation techniques that are readily available, inexpensive and easy to apply is vital to maintaining access.

Conservation should tackle a multitude of degradation processes arising from environmental factors, physical erosion and microorganisms, as well as handling and wrecking. Although the preservation of cultural heritage involves a different code of ethics ${ }^{2}$, it can be compared to medicine, where artefacts are analogous to patients and conservators are similar to doctors. Diagnosis, treatment and prevention are relevant to the conservation of artefacts, and science has contributed to such activities. Although much effort has been dedicated to preventive conservation and to the development of advanced diagnostic techniques, only a relatively small part of conservation science has focused on 'therapy' - that is, the production of innovative materials that can be applied to works of art to repair and restore them.

Nanoscience is a unique resource to conservation because, unlike conventional materials such as polymers that are commonly used in conservation, engineered nanomaterials do not alter the original physical and chemical properties of artefacts and have low environmental impact $^{3}$. Here, we highlight the role of hard (inorganic nanocrystals) and soft (built from molecular blocks) nanomaterials in revolutionizing the technical approaches to heritage conservation.

\section{Soft nanomaterials}

The first application of nanoscience to the conservation of artefacts dates back to the end of the 1980s in Florence, Italy, with the cleaning of wall paintings in the Brancacci Chapel ${ }^{4}$. Cleaning the surface of works of art is an irreversible and delicate intervention involving the removal of undesired materials layer by layer. In some cases, for instance on hard materials such as marble, stone and metal surfaces, this can be achieved using laser or plasma techniques ${ }^{5}$. However, these methods can induce local heating and mechanical shocks, particularly with painted surfaces. Chemical or wet methodologies become the preferred options because they are more practical and considered to be 'safer'.

a

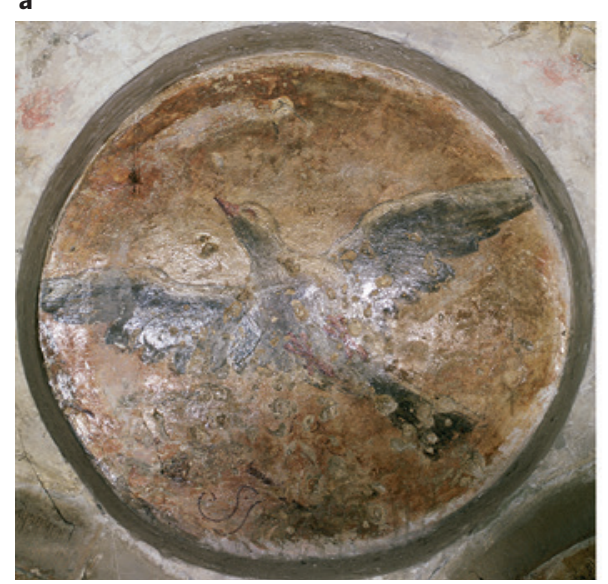

For the restoration of the Brancacci Chapel paintings, an oil-in-water microemulsion of dodecane nanodroplets stabilized in water by a surfactant was used to remove wax spots from the surface of the murals. As the amount of organic phase used in oil-in-water microemulsions is typically below $10 \%$ (including solvents and surfactants), their toxicity and environmental impact are significantly less than that of organic solvents (such as aliphatic and aromatic hydrocarbons) traditionally used for cleaning. Even with a reduced solvent content, microemulsions allow effective cleaning because the nanosize droplets have a huge interfacial area (that is, the active surface in soil removal) that is about 500,000 times higher than that of the same amount of bulk solvent. The soil detaches from the painting's surface and is trapped inside the droplets. Moreover, unlike bulk solvents, the continuous aqueous phase of the microemulsion acts as a barrier that limits the spreading of

\section{b}

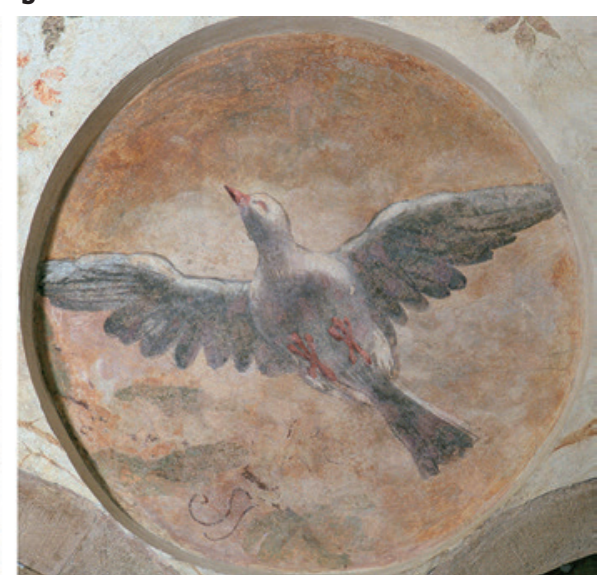

Figure 1 | Removal of aged acrylic coatings from the wall paintings of the San Salvador church sacristy in Venice, Italy. a,b, Photographs taken before the removal (a) and after the application of a high-viscosity polymeric dispersion loaded with an oil-in-water microemulsion (b). Panel a reproduced with permission from ref. 26, American Chemical Society. Panel $\mathbf{b}$ taken by Emiliano Carretti, printed with kind permission from the Italian Heritage Department. 

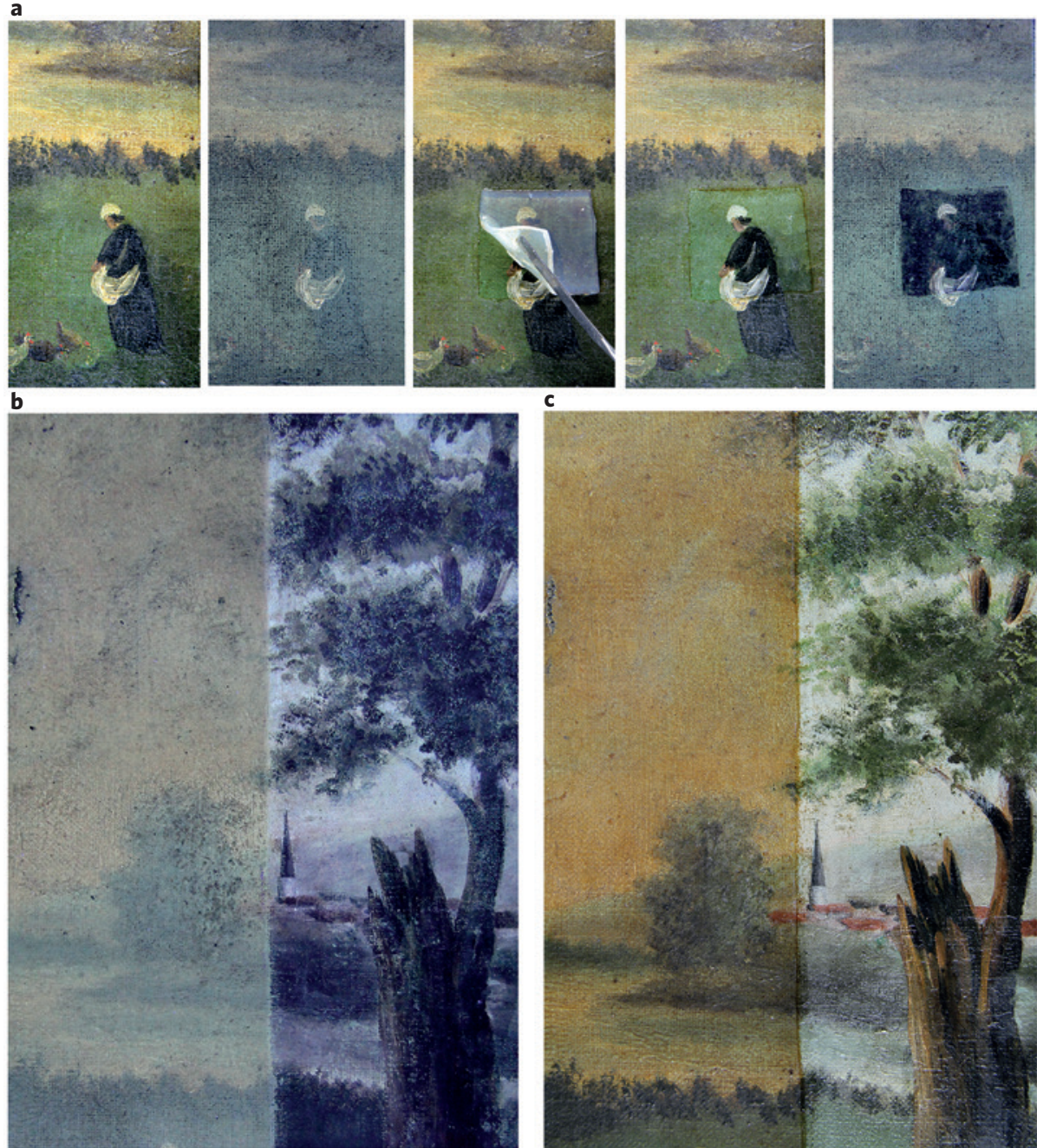

C

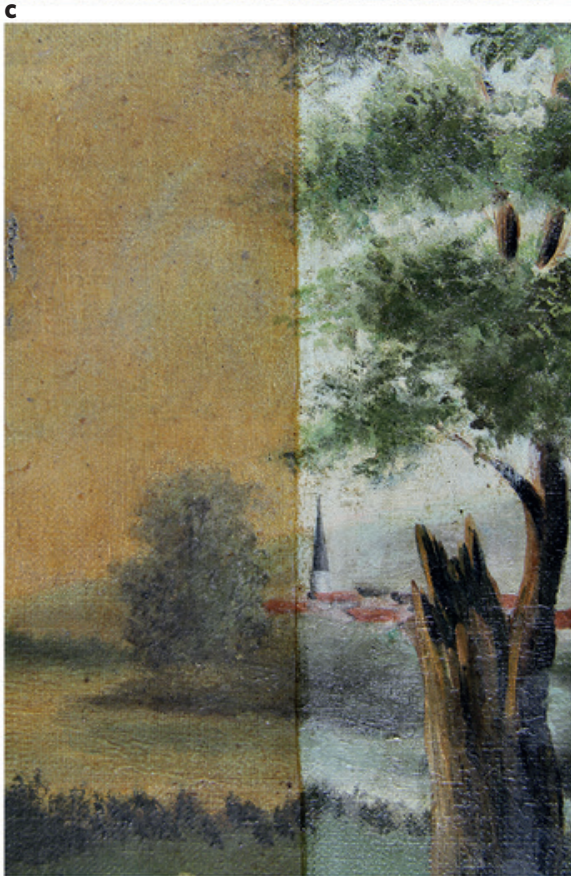

Figure 2 | Removal of aged varnish from an eighteenth-century canvas painting. a, Photographs of the painting and of the poly(2-hydroxyethyl methacrylate)/poly(vinylpyrrolidone) hydrogel application. From left to right: the painting before cleaning (visible light); the painting before cleaning (ultraviolet light); the application of the hydrogel (visible light); the painting after cleaning (visible light); and the painting after cleaning (ultraviolet light). b, Ultraviolet photographs of the painting showing the feasibility of using chemical gels over a large area. Ultraviolet light fluorescence highlights the efficacy of the cleaning process (left image, not cleaned; right image, cleaned). c, Visible light photographs of the painting showing the feasibility of using chemical gels over a large area (left image, not cleaned; right image, cleaned). Photographs courtesy of Nicole Bonelli, Michele Baglioni and Joana Domingues, CSGI. The painting was provided by Aurelia Chevalier, Atelier Chevalier, France.

the trapped wax into the pores of the wall painting. As a result more than $99 \%$ of the undesired materials are removed with a single application.

Since this pioneering application, several systems have been developed to address complex cleaning tasks, including those arising from previous detrimental restoration interventions. Since the 1960s, murals have been widely treated with hydrophobic polymer coatings because these materials, which are good adhesives, easy to use and offer a nice saturation of colours, were considered a panacea for many degradation issues ${ }^{6,7}$. However, these coatings strongly alter the surface permeability of the artefacts, resulting in mechanical stress that eventually produces flaking and detachment of the pictorial layer. Moreover, depending on the environmental conditions, these coatings can undergo degradation after $20-30$ years, and exhibit yellowing and brittleness. The removal of these polymers represents one of the most complex and ubiquitous topics in the conservation of cultural heritage.

A water-based amphiphilic formulation containing water, ethyl acetate, propylene carbonate, a surfactant and a cosurfactant was successfully used for the removal of aged acrylic-vinyl copolymers that were applied to Maya and Nahua murals in the archaeological sites of Mayapan and Cholula in Mexico $^{3}$. As the organic solvents (ethyl acetate and propylene carbonate) are present both in the continuous phase (water) and in the dispersed phase (nanocontainers of selfassembled amphiphiles), the system (named EAPC) is neither a classical microemulsion, such as that used in the Brancacci Chapel, nor a simple micellar solution. These features make EAPC highly effective: the nanocontainers allow the right amounts of organic solvent to be dispersed in water and made available for interaction with the aged acrylic-vinyl copolymer coating the murals. This interaction leads to swelling, chain disentanglement and eventual detachment of the copolymer.

EAPC has also been used to remove aged coatings (including silicone resins) from paintings in the Annunciation church in Nazareth, Israel, where traditional solvents (for example, a mixture of aliphatic and aromatic hydrocarbons) had proved ineffective ${ }^{8}$. A real improvement in nanostructured cleaning fluids is the use of surfactants that self-degrade to inert volatile compounds, making application even to fragile or sensitive painted surfaces possible, where rinsing with water or solvents to remove residues of surfactants might be detrimental ${ }^{9}$.

Furthermore, micelles and microemulsions can be confined into highly viscous gel-like matrices such as hydrophobically modified hydroxyethyl cellulose (hMHEC) or polyvinyl alcohol crosslinked with sodium tetraborate (PVA-borate polymeric dispersions) ${ }^{10,11}$. This combination allows spatial and kinetic control of the cleaning process. PVA-borate dispersions are highly elastic and they can be removed using tweezers. A hMHEC dispersion loaded with an oil-in-water microemulsion has been used to remove a thick layer of aged acrylic coatings from the surface of wall paintings in the San Salvador church in Venice (Fig. 1).

Another category of systems effective for the safe cleaning of painted surfaces is chemical gels whose gelling state is the result of covalent bond formation. Targeted and sustained drug delivery systems have inspired the development of new approaches for the controlled delivery of cleaning agents ${ }^{12}$. In particular, chemical gels made by semi-interpenetrated networks of poly(2-hydroxyethyl methacrylate) and poly(vinylpyrrolidone) can be shaped into thin transparent foils where oil-in-water microemulsions (and also polar solvents) 
can be easily loaded for the 'safe' removal of the soiling. The network of covalent bonds provides the gels with optimal mechanical properties allowing the removal of the foils after cleaning without leaving gel residues on the artefacts ${ }^{3}$. Moreover, the nano- and microporosity of the covalent network, and the hydrophilicity of the gels, can be tuned to be highly retentive, allowing a fine and controlled release of water-based cleaning fluids on water-sensitive objects (such as easel paintings, parchment and paper manuscripts, or dyed textiles), while preserving the high cleaning efficiency of microemulsions (Fig. 2).

\section{Hard nanomaterials}

When applied to the preservation of cultural heritage, nanotechnology also encompasses the use of dispersions of hard nanocrystals, such as inorganic hydroxides. At the end of the twentieth century, nanostructured calcium hydroxide $\left(\mathrm{Ca}(\mathrm{OH})_{2}\right)$ emerged as a highly beneficial restoration material because it can be used to strengthen weakened wall paintings and limestones ${ }^{13}$, and to neutralize acidity that degrades cellulose in wood and paper artefacts ${ }^{14,15}$.

Although highly insoluble compounds can easily be obtained on the nanoscale, the first attempt to synthesize moderately soluble nanoparticles (for example, $\mathrm{Ca}(\mathrm{OH})_{2}$, with solubility of around $10^{-5} \mathrm{M}$ ) took place in $1996^{13}$. Initially, $\mathrm{Ca}(\mathrm{OH})_{2}$ nanoplatelets (approximately $250 \mathrm{~nm}$ wide and $10 \mathrm{~nm}$ thick) were obtained through the co-precipitation of calcium chloride and sodium hydroxide solutions in water. Subsequently, several other synthetic methods have been investigated to improve the physicochemical properties of the particles, for instance to obtain smaller platelets (approximately $80 \mathrm{~nm}$ ) that exhibit enhanced penetration through porous artefacts and higher reactivity. Once inside the pores, $\mathrm{Ca}(\mathrm{OH})_{2}$ particles react with atmospheric $\mathrm{CO}_{2}$ (through the carbonation process) and transform into a new calcium carbonate network that merges with the carbonatic matrix constituting the painting to reproduce the mechanical properties of the original artefacts. Highly crystalline nanoparticles produce crystalline carbonate networks that are particularly resistant to mechanical stress and weather. Besides, the chemical reactivity of the nanomaterials and their ability to consolidate art works also depends on the environmental conditions under which carbonation takes place ${ }^{16,17}$. A relevant case study on this methodology is the consolidation of the Maya wall paintings in Calakmul, Mexico ${ }^{3}$ (Fig. 3).

$\mathrm{Ca}(\mathrm{OH})_{2}$ nanoparticles can also be used to recover the mechanical properties

$\mathbf{a}$
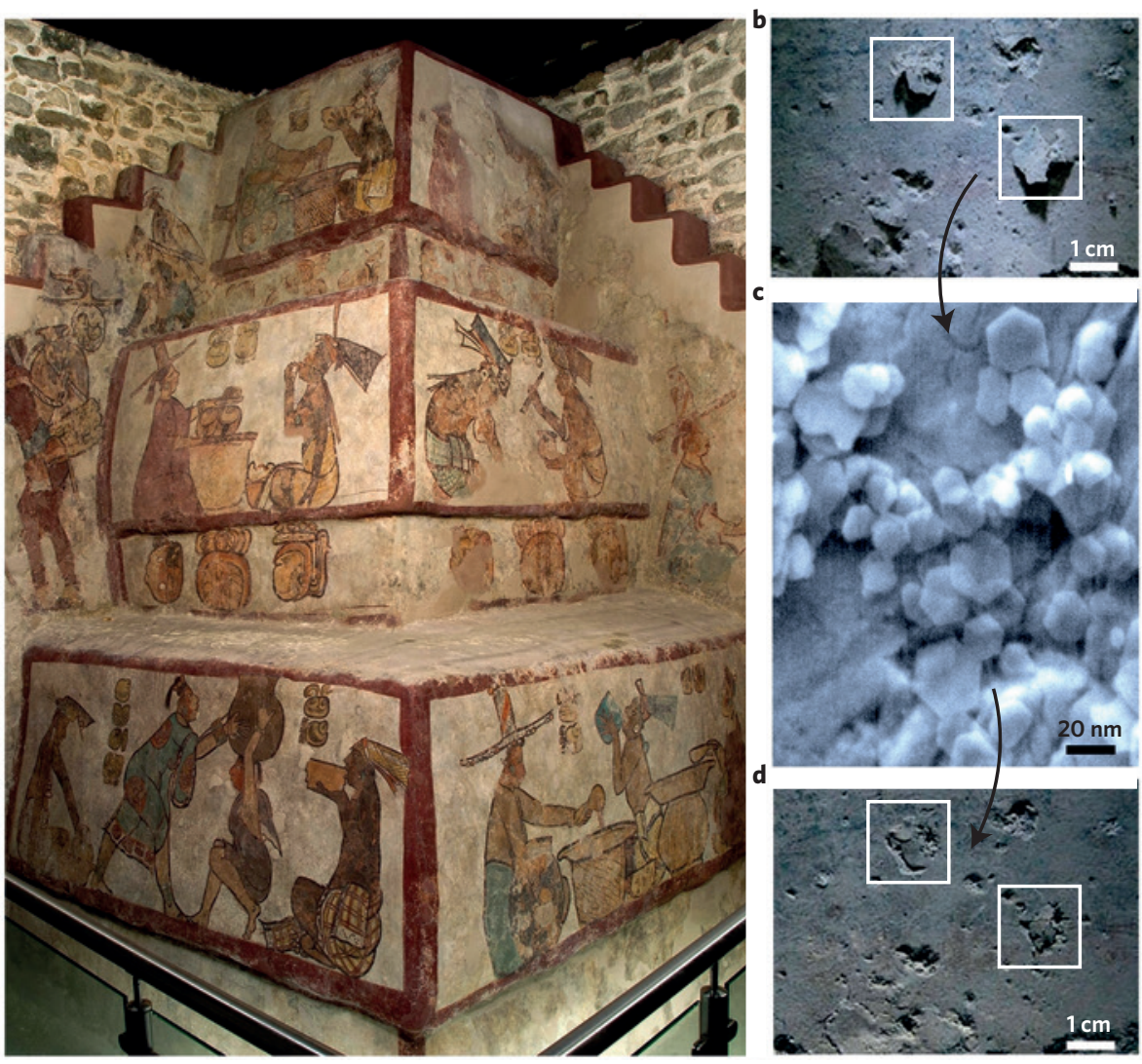

Figure 3 | Maya wall paintings in the United Nations Educational, Scientific and Cultural Organization world heritage site of Calakmul, Mexico. a, Photograph of the wall paintings after restoration with $\mathrm{Ca}(\mathrm{OH})_{2}$ nanoparticle dispersions. b, Grazing visible light image showing the detaching paint flakes before the application of nanoparticles. c, Scanning electron microscopy micrograph of the $\mathrm{Ca}(\mathrm{OH})_{2}$ nanoparticles (hexagonal platelets) that have been applied to the degraded painted surface. d, Grazing visible light image showing re-attached and re-adhered paint flakes after the application of nanoparticles. Panels a,b,d reproduced with permission from ref. 27, The Royal Society of Chemistry.

of archaeological and palaeontological bones, whose decay is based on a mechanism similar to osteoporosis. $\mathrm{Ca}(\mathrm{OH})_{2}$ nanoparticles penetrate the pores of the bones and react with atmospheric $\mathrm{CO}_{2}$ in the presence of trace amounts of magnesium ions and collagen residues. Magnesium ions and collagen favour the formation of aragonite, a crystalline form of calcium carbonate, with excellent mechanical properties ${ }^{18}$.

To address conservation issues, it is necessary to obtain stable and well-dispersed $\mathrm{Ca}(\mathrm{OH})_{2}$ nanoparticles in non-aqueous solvent, because water can cause hydrophilic layers on the artwork to swell and/or dyes to leach out. This requirement prompted the application of nanoparticles that are stably dispersed in short-chain alcohols ${ }^{13}$. The use of these solvents on water-sensitive components (for example, most ancient inks) is less risky and advantageous because they prevent the agglomeration of particles into bigger clusters without the need for additives or stabilizers; this allows particles to penetrate better and more homogeneously through the artefact. In the past decade, nanoparticle dispersions in alcohols have been widely assessed by conservators and conservation scientists as feasible tools to effectively improve the mechanical resistance of weakened murals, and to safely counteract the acidity of different artefacts including historical inked paper documents, parchment, the canvas support of paintings and waterlogged wood coming from shipwrecks ${ }^{3}$.

The consolidation of silicate-based stones has also benefitted from the use of nanomaterials. The main inconvenience of using well-established commercial consolidation products (that is, ethyl orthosilicate solutions) is that, on drying, they tend to crack inside the pores of the stone inducing mechanical stresses on the silicate matrix ${ }^{19}$. By adding silica nanoparticles, flexible silanes or nanometresized polyhedral oligomeric silsesquioxane 
to ethyl orthosilicate solutions, crack formation during the drying phase is reduced ${ }^{20,21}$.

Nanosols of silica have recently been used in the consolidation of wood artefacts and prevention of dimensional instability owing to swelling and shrinkage induced by changes in temperature and relative humidity ${ }^{22,23}$. Nanosols functionalized with organic groups (specifically alkoxysilanes) can stably bind to wood through the formation of covalent bonds with hydroxyl groups of wood cellulose. The equilibration moisture of wood decreases following treatment with sols, leading to dimensional stability. Furthermore, alkyl-modified silica sols increase the abrasion resistance of wood.

Although nanomaterials offer new opportunities for the conservation of cultural heritage, the environmental and health concerns surrounding their use must be critically addressed. This is the case, for instance, for titanium dioxide $\left(\mathrm{TiO}_{2}\right)$ nanoparticles, which can be used as coatings for stone surfaces and can also be embedded into a high-performance concrete to achieve a surface active against pollution and microbial contamination. The first example of such an architectonic building designed with a self-cleaning white surface is Meier's church, Dives in Misericordia, in Rome. Recently, the need to assess the durability and sustainability of $\mathrm{TiO}_{2}$ nanoparticles through a life cycle assessment methodology was highlighted that is, a complete and exhaustive evaluation that considers both performance and environmental impact during all stages of the material's life (from production to post-application phases) ${ }^{24}$. At present, this practice has not yet been routinely adopted for nanomaterials used in cultural heritage conservation.

In some cases, ageing in the artwork can cause the release of nanoparticles into the environment. One way to inhibit this release is by binding the nanoparticles stably to the treated artwork. For example, chemically modified silver nanoparticles bound to a bifunctional molecule (such as one consisting of silanes and alkyl orthosilicates bearing a short hydrocarbon chain) have been stably grafted onto stone surfaces ${ }^{25}$. In addition to providing stability, preliminary experiments have shown that grafting silver nanoparticles can prevent bacterial and fungal contamination ${ }^{25}$.

\section{Call to material scientists}

We expect several challenges in the next few decades. Although the nanomaterials developed so far are able to conserve the older legacies, new applications must be explored to safely preserve modern and contemporary art for future generations. As contemporary artefacts (for example, plastic sculptures, polymateric artworks, inked drawings) degrade very rapidly, it is expected that many of these important works of art may be severely damaged within the next 50 years. Such an urgent and concerning threat stands as a call to material scientists to participate in cultural heritage conservation and to develop innovative materials for the preservation of our cultural identity.

Piero Baglioni, Emiliano Carretti and David Chelazzi are in the Department of Chemistry and Center for Colloids and Surface Science, University of Florence, via della Lastruccia, 3 - Sesto Fiorentino, 50019 Florence, Italy. e-mail:piero.baglioni@unifi.it
References

1. Dümcke, C. \& Gnedovsky, M. The Social and Economic Value of Cultural Heritage: Literature Review EENC Paper (EENC, July 2013); available via http://go.nature.com/x57jKP

2. ECCO Code of Ethics http://www.ecco-eu.org/about-e.c.c.o./ professional-guidelines.html (ECCO, 2003).

3. Baglioni, P. \& Chelazzi, D. (eds) Nanoscience for the Conservation of Works of Art (RSC Nanoscience \& Nanotechnology series 28, The Royal Society of Chemistry, 2013).

4. Baglioni, P. et al. Adv. Colloid Interface Sci. 205, 361-371 (2014).

5. Nevin, A., Pouli, P., Georgiou, S. \& Fotakis, C. Nature Mater. 6, 320-322 (2007).

6. Horie, C. V. Materials for Conservation (Butterworths, 1987)

7. Favaro, M. et al. Polym. Degrad. Stab. 91, 3083-3096 (2006)

8. Baglioni, M., Berti, D., Teixeira, J., Giorgi, R. \& Baglioni, P. Langmuir 28, 15193-15202 (2012).

9. Baglioni, M. et al. Soft Matter 10, 6798-6809 (2014).

10. Carretti, E., Fratini, E., Berti, E., Dei, L \& Baglioni, P. Angew. Chem. Int. Ed. 48, 8966-8969 (2009).

11. Carretti, E. et al. Acc. Chem. Res. 43, 751-760 (2010).

12. Wang, J., Sun, F. \& Li, X. J. Appl. Polym. Sci. 117, 1851-1858 (2010).

13. Ambrosi, M., Dei, L., Giorgi, R., Neto, C. \& Baglioni, P. Langmuir 17, 4251-4255 (2001).

14. Wouters, J. Science 322, 1196-1198 (2008).

15. Giorgi, R., Chelazzi, D. \& Baglioni, P. Langmuir 21, 10743-10748 (2005).

16. Rodriguez-Navarro, C., Ruis-Agudo, E., Ortega-Huertas, M. \& Hansen, E. Langmuir 21, 10948-10957 (2005).

17. López-Arcé, P. et al. Mater. Charact. 61, 168-184 (2010).

18. Natali, I. et al. Langmuir 30, 660-668 (2014).

19. Brus, J. \& Kotlik, P. Stud. Conserv. 41, 55-59 (1997).

20. Kim, E. K., Won, J., Do, J., Kim, S. D. \& Kang, Y. S. J. Cult. Herit. 10, 214-221 (2009).

21. Son, S. et al. Appl. Mater. Interfaces 1, 393-401 (2009).

22. Mahltig, B., Swaboda, C., Roessler, A. \& Böttcher, H. J. Mater. Chem. 18, 3180-3192 (2008).

23. Tingaut, P., Weigenand, O., Mai, C., Militz, H. \& Sebe, G. Holzforschung 60, 271-277 (2006)

24. Munafò, P., Quagliarini, E., Goffredo, G. B., Bondioli, F. \& Licciulli, A. Constr. Build. Mater. 65, 218-231 (2014)

25. Bellissima, F. et al. Environ. Sci. Pollut. Res. 21, 13278-13286 (2014).

26. Carretti, E. et al. Langmuir 23, 6396-6403 (2007).

27. Baglioni, P., Carrasco Vargas, R., Cordeiro Baqueiro, M. \& Chelazzi, D. in Science and Art: The Painted Surface (eds Sgamellotti, A., Giovanni, B. G. \& Miliani, C.) Ch. 4, 68-93 (Royal Society of Chemistry, 2014).

Acknowledgements

CSGI and the European Union (NANOFORART project FP7-ENV-NMP-2011/282816; www.nanoforart.eu) are gratefully acknowledged for financial support. L. Dei, D. Berti, E. Fratini and R. GIorgi are gratefully acknowledged for their collaboration and discussions. 